\title{
Fuzzy Stochastic Vibrations of Double-Beam Complex System as Model Sandwich Beam with Uncertain Parameters
}

\author{
Krystyna Mazur-Śniady, ${ }^{1}$ Katarzyna Misiurek, ${ }^{1}$ Olga Szyłko-Bigus, ${ }^{1}$ and Paweł Śniady ${ }^{2}$ \\ ${ }^{1}$ Wroclaw University of Technology, Institute of Civil Engineering, Wybrzeże Wyspiańskiego 27, 50-370 Wrocław, Poland \\ ${ }^{2}$ Wroclaw University of Environmental and Life Science, The Faculty of Environmental Engineering and Geodesy, \\ Plac Grunwaldzki 24, 50-365 Wroclaw, Poland
}

Correspondence should be addressed to Paweł Śniady; pawel.sniady@wp.pl

Received 31 July 2013; Accepted 18 August 2013

Academic Editors: S. He, Y. Wang, and X. Xue

Copyright ( $) 2013$ Krystyna Mazur-Śniady et al. This is an open access article distributed under the Creative Commons Attribution License, which permits unrestricted use, distribution, and reproduction in any medium, provided the original work is properly cited.

The dynamic behavior of a double Euler-Bernoulli beam system with uncertain parameters (fuzzy random variables) under a fuzzy stochastic excitation and axial compression is being considered. The beams are identical and parallel, one is above the other, and they are continuously coupled by a linear two-parameter (Pasternak subsoil) elastic element. This double Euler-Bernoulli beam system can be also treated as a theoretical model of a sandwich beam. The load process is fuzzy random both in space and time. The top beam carries a fuzzy stochastic load. The solution of the problem was found thanks to the fuzzy random dynamic influence function. The aim of the paper is to find the solution for the membership function of the probabilistic characteristics of the response of the structure.

\section{Introduction}

Uncertainty modeling in computational mechanics has received significant attention in recent years. Dynamic analysis of structures often involves two kinds of uncertainty. One of them is referred to as aleatory uncertainty (randomness, stochastic variability) and the other as epistemic uncertainty which describes, among others, imprecision, vagueness, ambiguity, and lack of the data. The random variability is described by the use of the probability theory and the imprecision by the use of fuzzy sets. Very often sufficient statistical data is not available; in this case a fuzzy function (fuzzy process) or a fuzzy random variable (fuzzy stochastic process) is suitable for the modeling purposes. The basis of the probabilistic methods is primarily statistical data while in the theory of fuzzy sets we refer also to the human intuition and experience. Using fuzzy random variables or fuzzy stochastic processes we combine these two approaches. The classical problem of the dynamic response of structures under stochastic excitation has been presented in many monographs. In most cases it is assumed that the parameters of the structure are deterministic. On the other hand, the structural parameters like geometry characteristics and material and damping properties might be uncertain to some extent. Their uncertainty may have a strong influence on the reliability of a structure in the dynamic context. The idea of fuzzy set theory was initiated by Zadeh [1]. The concept of fuzzy random variables was introduced by Kwakernaak [2] and Puri and Ralescu [3] and combines both randomness and imprecision. The application of the uncertain forecasting in engineering and computational mechanics based on fuzzy stochastic processes is presented in the monograph [4]. Various types of structures and girders like beams, plates, shells, and frames have been considered. An important technological extension of a single string, beam, or plate is a double-string, doublebeam, or string-beam system. Various aspects of the dynamic response of a double-string and double-beam system have been considered by Oniszczuk [5-9]. Free and forced vibrations of a double-beam system have been considered among others in $[10,11]$. The problem of the vibration and buckling of a double-beam system under compressive axial loading is presented in [12]. Vibrations of a complex system under moving force have been studied in [13-15].

In this paper the dynamic behavior of a double EulerBernoulli beam system with uncertain parameters (fuzzy random variables) under a fuzzy stochastic excitation and axial 
compression is being considered. The beams are identical, parallel, one above the other and continuously coupled by a linear two-parameter (Pasternak subsoil) elastic element. This double Euler-Bernoulli beam system can be also treated as a theoretical model of a sandwich beam, in which the shear and compression of core are taken into account. The load process is fuzzy random both in space and time. The solution of the problem was found thanks to the fuzzy random dynamic influence function [16-18]. The aim of the paper is to find the solution for the membership function of the probabilistic characteristics of the response of the structure. The probabilistic characteristics of the response of the structure are sought in the form of the first two probabilistic moments, that is, the expected value and the correlation (covariance) function. The stochastic response of structure with random and uncertain parameters has been considered, among others, in [18-27]. Fuzzy stochastic finite element method based a spectral approach to analyze complex engineering structures under dynamic excitation has been presented in [28].

\section{Formulation of the Problem: General Solution}

Let us consider the vibrations of a connected double EulerBernoulli beam continuous system connected by two-parameters elastic element under axial compression $N$ excited as is shown Figure 1.

The differential equations of motion of the beams system have the form

$$
\begin{aligned}
& E_{0} I \frac{\partial^{4} w_{1}(x, t)}{\partial x^{4}}+N \frac{\partial^{2} w_{1}(x, t)}{\partial x^{2}} \\
& -k_{1}\left[\frac{\partial^{2} w_{1}(x, t)}{\partial x^{2}}-\frac{\partial^{2} w_{2}(x, t)}{\partial x^{2}}\right] \\
& +k_{0}\left[w_{1}(x, t)-w_{2}(x, t)\right] \\
& +c \frac{\partial w_{1}(x, t)}{\partial t}+m \frac{\partial^{2} w_{1}(x, t)}{\partial t^{2}}=p(x, t) \\
& E_{0} I \frac{\partial^{4} w_{2}(x, t)}{\partial x^{4}}+N \frac{\partial^{2} w_{2}(x, t)}{\partial x^{2}} \\
& -k_{1}\left[\frac{\partial^{2} w_{2}(x, t)}{\partial x^{2}}-\frac{\partial^{2} w_{1}(x, t)}{\partial x^{2}}\right] \\
& +k_{0}\left[w_{2}(x, t)-w_{1}(x, t)\right] \\
& +c \frac{\partial w_{2}(x, t)}{\partial t}+m \frac{\partial^{2} w_{2}(x, t)}{\partial t^{2}}=0
\end{aligned}
$$

where $E_{0} I$ is the flexural rigidity of the beam, $E_{0}$ is Young's modulus of elasticity, $I$ is the moment of inertia of the crosssection area of the beam, $m$ is the mass of the beam, $c$ is the damping coefficient, $k_{0}$ is the elastic stiffness (Winkler's parameter), and $k_{1}$ is the shear stiffness of the elastic element.

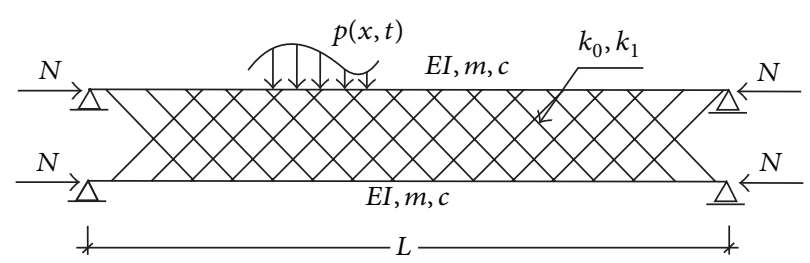

FIgURE 1: Double-beam system.

The boundary conditions of the simply supported beam are

$$
\begin{aligned}
w_{1}(0, t) & =w_{1}(L, t)=0, \\
\left.\frac{\partial^{2} w_{1}(x, t)}{\partial x^{2}}\right|_{x=0} & =\left.\frac{\partial^{2} w_{1}(x, t)}{\partial x^{2}}\right|_{x=L}=0, \\
w_{2}(x, 0) & =w_{2}(x, L)=0, \\
\left.\frac{\partial^{2} w_{2}(x, t)}{\partial x^{2}}\right|_{x=0} & =\left.\frac{\partial^{2} w_{2}(x, t)}{\partial x^{2}}\right|_{x=L}=0 .
\end{aligned}
$$

The above double-beam complex system can be treated also as a theoretical model of a sandwich beam which consists of two bendable outer layers and a sheared middle layer, which is also compressed.

The following system parameters are assumed to be uncertain: the Young's modulus $E_{0}=E_{0 \alpha}(e)$, the mass of the beam $m=m_{\alpha}(e)$, the damping coefficient $c=c_{\alpha}(e)$, and the parameters of the elastic element $k_{0}=k_{0 \alpha}(e), k_{1}=$ $k_{1 \alpha}(e)$. The symbol $e$ denotes an elementary event, and for simplicity we will skip it in the paper. The symbol $\alpha$ denotes the fuzziness of the parameters. The uncertain parameters of the system are assumed to be fuzzy random variables. The load process $p(x, t)=p_{\alpha}(e, x, t)$ is assumed to be a fuzzy stochastic process. The responses of the system $w_{1}(x, t)=$ $w_{1 \alpha}(e, x, t)$ and $w_{2}(x, t)=w_{2 \alpha}(e, x, t)$ are also fuzzy stochastic processes as well. The aim is to find the solution for the membership function of the probabilistic characteristics of the response, $w_{1 \alpha}(x, t)$ and $w_{2 \alpha}(x, t)$ of the double-beam complex system. The probabilistic characteristics of the response of the structure are sought in the form of the first two probabilistic moments, that is, the expected value and the correlation (covariance) function.

In order to decouple (1) and (2), let us introduce two new functions

$$
\begin{gathered}
w_{I \alpha}(x, t)=w_{1 \alpha}(x, t)+w_{2 \alpha}(x, t), \\
w_{I I \alpha}(x, t)=w_{1 \alpha}(x, t)-w_{2 \alpha}(x, t) .
\end{gathered}
$$

From (1) and (2), we obtain two new differential equations for functions $w_{I \alpha}(x, t)$ and $w_{I I \alpha}(x, t)$ :

$$
\begin{gathered}
E_{0 \alpha} I \frac{\partial^{4} w_{I \alpha}(x, t)}{\partial x^{4}}+N \frac{\partial^{2} w_{I \alpha}(x, t)}{\partial x^{2}}+c_{\alpha} \frac{\partial w_{I \alpha}(x, t)}{\partial t} \\
+m_{\alpha} \frac{\partial^{2} w_{I \alpha}(x, t)}{\partial t^{2}}=p_{\alpha}(x, t),
\end{gathered}
$$




$$
\begin{aligned}
E_{0 \alpha} I & \frac{\partial^{4} w_{I I \alpha}(x, t)}{\partial x^{4}}+\left(N-2 k_{1 \alpha}\right) \frac{\partial^{2} w_{I I \alpha}(x, t)}{\partial x^{2}} \\
& +2 k_{0 \alpha} w_{I I \alpha}(x, t)+c_{\alpha} \frac{\partial w_{I I \alpha}(x, t)}{\partial t}+m_{\alpha} \frac{\partial^{2} w_{I I \alpha}(x, t)}{\partial t^{2}} \\
= & p_{\alpha}(x, t) .
\end{aligned}
$$

Equation (5) describes vibrations of a single beam, while (6) describes vibrations of a single beam resting on an elastic Pasternak support with parameters $2 k_{0}$ and $2 k_{1}$. From (4), it follows that

$$
\begin{aligned}
& w_{1 \alpha}(x, t)=\frac{w_{I \alpha}(x, t)+w_{I I \alpha}(x, t)}{2}, \\
& w_{2 \alpha}(x, t)=\frac{w_{I \alpha}(x, t)-w_{I I \alpha}(x, t)}{2} .
\end{aligned}
$$

In the case when the parameters of (5) and (6) are random, we can solve the problem if the right-hand side is deterministic. To overcome these difficulties, we introduce the space-time fuzzy random dynamic influence functions $H_{I \alpha}(x, t)$ and $H_{I I \alpha}(x, t)$ (FRDIF) which satisfy the following equations with a deterministic right-hand side:

$$
\begin{aligned}
E_{0 \alpha} I & \frac{\partial^{4} H_{I \alpha}(x, \xi, t)}{\partial x^{4}}+N \frac{\partial^{2} H_{I \alpha}(x, \xi, t)}{\partial x^{2}}+c_{\alpha} \frac{\partial H_{I \alpha}(x, \xi, t)}{\partial t} \\
& +m_{\alpha} \frac{\partial^{2} H_{I \alpha}(x, \xi, t)}{\partial t^{2}}=\delta(t) \delta(x-\xi), \\
E_{0 \alpha} I & \frac{\partial^{4} H_{I I \alpha}(x, \xi, t)}{\partial x^{4}}+\left(N-2 k_{1 \alpha}\right) \frac{\partial^{2} H_{I I \alpha}(x, \xi, t)}{\partial x^{2}} \\
& +2 k_{0 \alpha} H_{I I \alpha}(x, \xi, t)+c_{\alpha} \frac{\partial H_{I I \alpha}(x, \xi, t)}{\partial t} \\
& +m_{\alpha} \frac{\partial^{2} H_{I I \alpha}(x, \xi, t)}{\partial t^{2}}=\delta(t) \delta(x-\xi) .
\end{aligned}
$$

After solving (8) one obtains

$$
\begin{aligned}
& H_{1 \alpha}(x, \xi, t)=\frac{H_{I \alpha}(x, \xi, t)+H_{I I \alpha}(x, \xi, t)}{2}, \\
& H_{2 \alpha}(x, \xi, t)=\frac{H_{I \alpha}(x, \xi, t)-H_{I I \alpha}(x, \xi, t)}{2} .
\end{aligned}
$$

If the FRDIF $H_{1 \alpha}(x, \xi, t)$ and $H_{2 \alpha}(x, \xi, t)$ are known, the response of the system $w_{1 \alpha}(x, t)$ and $w_{2 \alpha}(x, t)$ can be presented in the following form:

$$
w_{i \alpha}(x, t)=\int_{0}^{L} \int_{t_{0}}^{t} H_{i \alpha}(x, \xi, t-\tau) p_{\alpha}(\xi, \tau) d \tau d \xi,
$$

where $i=1,2$, and if $t_{0}=0$, then one considers transition vibrations, and for $t_{0}=-\infty$, one considers steady-state vibration case.

Thus, in order to determine the probabilistic characteristics of the displacement of the beams, one can apply the expectancy operator to (10) and consequently obtain the expected values as follows:

$$
\begin{aligned}
& E\left[w_{i \alpha}(x, t)\right]_{\alpha} \\
& \quad=\int_{0}^{L} \int_{t_{0}}^{t} E\left[H_{i \alpha}(x, \xi, t-\tau)\right] E\left[p_{\alpha}(\xi, \tau)\right] d \tau d \xi .
\end{aligned}
$$

Using $\alpha$-level optimization procedure [4] for arbitrary $\alpha=$ $\alpha_{k} \in[0,1]$ or the max-min operator in the extension principle [1], the smallest and the largest expected values at an established point $x$ and time $t$ can be found.

Due to relationship (11), one obtains

$$
\begin{array}{r}
E\left[w_{i \alpha}(x, t)\right]_{\alpha l} \\
=\min \left\{\int_{0}^{L} \int_{t_{0}}^{t} E\left[H_{i \alpha}(x, \xi, t-\tau)\right] E\left[p_{\alpha}(\xi, \tau)\right] d \tau d \xi\right\}, \\
E\left[w_{i \alpha}(x, t)\right]_{\alpha r} \\
=\max \left\{\int_{0}^{L} \int_{t_{0}}^{t} E\left[H_{i \alpha}(x, \xi, t-\tau)\right] E\left[p_{\alpha}(\xi, \tau)\right] d \tau d \xi\right\} .
\end{array}
$$

Taking into account (10), the covariance functions of the beams displacement on the fuzziness level $\alpha$ have the form

$$
\begin{array}{rl}
C_{w_{i \alpha}}\left(x_{1}, x_{2}, t_{1}, t_{2}\right) & \\
=\int_{0}^{L} \int_{0}^{L} \int_{t_{0}}^{t_{1}} \int_{t_{0}}^{t_{2}} & E\left[H_{i \alpha}\left(x_{1}, \xi_{1}, t_{1}-\tau_{1}\right) H_{i \alpha}\left(x_{2}, \xi_{2}, t_{2}-\tau_{2}\right)\right] \\
\times & C_{(p p)_{\alpha}}\left(\xi_{1}, \xi_{2}, \tau_{1}, \tau_{2}\right) d \tau_{1} d \tau_{2} d \xi_{1} d \xi_{2} \\
+\int_{0}^{L} \int_{0}^{L} \int_{t_{0}}^{t_{1}} \int_{t_{0}}^{t_{2}} C_{\left(H_{i} H_{i}\right)_{\alpha}}\left(x_{1}, x_{2}, \xi_{1}, \xi_{2}, t_{1}-\tau_{1}, t_{2}-\tau_{2}\right) \\
\times E\left[p_{\alpha}\left(\xi_{1}, \tau_{1}\right)\right] \\
\times E\left[p_{\alpha}\left(\xi_{2}, \tau_{2}\right)\right] d \tau_{1} d \tau_{2} d \xi_{1} d \xi_{2},
\end{array}
$$

where

$$
\begin{aligned}
C_{\left(H_{i} H_{j}\right)_{\alpha}}\left(x_{1}, x_{2}, \xi_{1}, \xi_{2}, t_{1}, t_{2}\right) \\
=E\left[H_{i \alpha}\left(x_{1}, \xi_{1}, t_{1}\right) H_{j \alpha}\left(x_{2}, \xi_{2}, t_{2}\right)\right]_{\alpha} \\
\quad-E\left[H_{i \alpha}\left(x_{1}, \xi_{1}, t_{1}\right)\right]_{\alpha} E\left[H_{j \alpha}\left(x_{2}, \xi_{2}, t_{2}\right)\right]_{\alpha}, \\
C_{(p p)_{\alpha}}\left(\xi_{1}, \xi_{2}, \tau_{1}, \tau_{2}\right) \\
=E\left[p_{\alpha}\left(\xi_{1}, \tau_{1}\right) p_{\alpha}\left(\xi_{2}, \tau_{2}\right)\right]_{\alpha} \\
\quad-E\left[p_{\alpha}\left(\xi_{1}, \tau_{1}\right)\right]_{\alpha} E\left[p_{\alpha}\left(\xi_{2}, \tau_{2}\right)\right]_{\alpha} .
\end{aligned}
$$


The lower and upper endpoints of the covariance could be defined using (13) as

$$
\begin{aligned}
C_{w_{i \alpha l}}\left(x_{1}, x_{2}, t_{1}, t_{2}\right) & \\
=\min \left\{\int_{0}^{L} \int_{0}^{L} \int_{t_{0}}^{t_{1}} \int_{t_{0}}^{t_{2}} E[\right. & H_{i \alpha}\left(x_{1}, \xi_{1}, t_{1}-\tau_{1}\right) \\
& \left.\times H_{i \alpha}\left(x_{2}, \xi_{2}, t_{2}-\tau_{2}\right)\right] C_{(p p)_{\alpha}} \\
\times & \left(\xi_{1}, \xi_{2}, \tau_{1}, \tau_{2}\right) d \tau_{1} d \tau_{2} d \xi_{1} d \xi_{2}
\end{aligned}
$$

$$
\begin{aligned}
& C_{w_{i \alpha r}}\left(x_{1}, x_{2}, t_{1}, t_{2}\right) \\
&=\max \left\{\int_{0}^{L} \int_{0}^{L} \int_{t_{0}}^{t_{1}} \int_{t_{0}}^{t_{2}} E\right. {\left[H_{i \alpha}\left(x_{1}, \xi_{1}, t_{1}-\tau_{1}\right)\right.} \\
&\left.\times H_{i \alpha}\left(x_{2}, \xi_{2}, t_{2}-\tau_{2}\right)\right] C_{(p p)_{\alpha}} \\
& \times\left(\xi_{1}, \xi_{2}, \tau_{1}, \tau_{2}\right) d \tau_{1} d \tau_{2} d \xi_{1} d \xi_{2}
\end{aligned}
$$

In order to find the expected values $E\left[H_{i \alpha}(x, \xi, t)\right]$ and $E\left[H_{i \alpha}\left(x_{1}, \xi_{1}, t_{1}\right) H_{i \alpha}\left(x_{2}, \xi_{2}, t_{2}\right)\right]$, we can use the perturbation method or Monte Carlo method. In this paper the perturbation method has been used for a particular solution of the expected values and variances and is presented in Section 3 for the response of the double-beam system.

\section{Particular Solutions}

The solutions of (8) for boundary conditions (3) are assumed to be in the form of the sine series:

$$
H_{J \alpha}(x, \xi, t)=\sum_{n=1}^{\infty} y_{J n \alpha}(t, \xi) \sin \frac{n \pi x}{L},
$$

where $J=I, I I$.

By substituting expression (17) into (8) and using orthogonalization method, one obtains the following set of uncoupled ordinary differential equations:

$$
\begin{aligned}
& \ddot{y}_{J n \alpha}(t, \xi)+2 \beta_{\alpha} \dot{y}_{J n \alpha}(t, \xi)+\omega_{J n \alpha}^{2} y_{J n \alpha}(t, \xi) \\
& =\frac{2}{m L} \delta(t) \sin \frac{n \pi \xi}{L}
\end{aligned}
$$

where, for $J=I, \omega_{\text {In } \alpha}^{2}=\omega_{\text {In } \alpha}^{2}=(n \pi / L)^{2}\left[E_{0 \alpha} I(n \pi / L)^{2}-N\right] / m$ and for $J=I I, \omega_{\text {In } \alpha}^{2}=\omega_{I I n \alpha}^{2}=\left((n \pi / L)^{4} E_{0 \alpha} I-(n \pi / L)^{2}(N-\right.$ $\left.\left.2 k_{1 \alpha}\right)+2 k_{0 \alpha}\right) / m$ and $2 \beta_{\alpha}=c_{\alpha} / m_{\alpha}$.

The dots denote differentiation with respect to the time. These functions fulfill the initial conditions

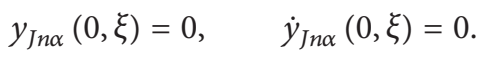

The solution of (18) taking into account the initial conditions (19) has the form

$$
y_{J n \alpha}(t, \xi)=\frac{2}{m L \bar{\omega}_{J n \alpha}} e^{-\beta_{\alpha} t} \sin \bar{\omega}_{J n \alpha} t \sin \frac{n \pi \xi}{L},
$$

where $\bar{\omega}_{\text {In } \alpha}^{2}=\omega_{\text {In } \alpha}^{2}-\beta_{\alpha}^{2}$.

Taking into account (9), (17), and (20) we have

$$
\begin{aligned}
& H_{1 \alpha}(x, \xi, t) \\
& \quad=\frac{1}{m L} e^{-\beta_{\alpha} t} \sum_{n=1}^{\infty}\left(\frac{\sin \bar{\omega}_{I n \alpha} t}{\bar{\omega}_{I n \alpha}}+\frac{\sin \bar{\omega}_{I I n \alpha} t}{\bar{\omega}_{I I n \alpha}}\right) \sin \frac{n \pi \xi}{L} \sin \frac{n \pi x}{L}, \\
& H_{2 \alpha}(x, \xi, t) \\
& =\frac{1}{m L} e^{-\beta_{\alpha} t} \sum_{n=1}^{\infty}\left(\frac{\sin \bar{\omega}_{I n \alpha} t}{\bar{\omega}_{I n \alpha}}-\frac{\sin \bar{\omega}_{I I n \alpha} t}{\bar{\omega}_{I I n \alpha}}\right) \sin \frac{n \pi \xi}{L} \sin \frac{n \pi x}{L} .
\end{aligned}
$$

Let us consider the steady-state vibration $\left(t_{0}=-\infty\right)$, assuming that the excitation load is a fuzzy weakly stationary "white noise" stochastic process both in time and space. In this case we have $E\left[p_{\alpha}(x, t)\right]_{\alpha}=E\left[p_{\alpha}\right]_{\alpha}=$ const. and $C_{(p p)_{\alpha}}\left(x_{1}, x_{2}, t_{1}, t_{2}\right)=\sigma_{(p) \alpha}^{2} \delta\left(t_{1}-t_{2}\right) \delta\left(x_{1}-x_{2}\right)$. We assume that the Young's modulus $E_{0 \alpha}$ is a fuzzy random variable. The other system parameters are assumed to be deterministic. In order to find the probabilistic characteristics the function of the random variables has been expanded into Taylor series around the mean value and restricted to three items of the expansion. The expected value for steady-state case is equal to

$$
\begin{aligned}
& E\left[w_{1 \alpha}(x, \infty)\right] \\
& =\frac{E\left[p_{\alpha}\right]_{\alpha}}{L} \sum_{n=1}^{\infty}\left(\frac{1-(-1)^{n}}{n \pi / L}\right)
\end{aligned}
$$




$$
\begin{aligned}
& \times\left\{\frac{1}{E\left[E_{0 \alpha}\right] I(n \pi / L)^{4}-N(n \pi / L)^{2}}\right. \\
& \times\left[1+\frac{(n \pi / L)^{8} I^{2} \sigma_{E_{0}}^{2}}{\left\{E\left[E_{0 \alpha}\right] I(n \pi / L)^{4}-N(n \pi / L)^{2}\right\}^{2}}\right] \\
& +\frac{1}{E\left[E_{0 \alpha}\right] I(n \pi / L)^{4}-\left(N-2 k_{1}\right)(n \pi / L)^{2}+2 k_{0}} \\
& \times\left[1+\left(\left(\frac{n \pi}{L}\right)^{8} I^{2} \sigma_{E_{0}}^{2}\right)\right. \\
& \quad \times\left(\left\{E\left[E_{0 \alpha}\right] I\left(\frac{n \pi}{L}\right)^{4}-\left(N-2 k_{1}\right)\left(\frac{n \pi}{L}\right)^{2}\right.\right. \\
& \left.\left.\left.\left.\quad+2 k_{0}\right\}^{2}\right)^{-1}\right]\right\} \sin \frac{n \pi x}{L},
\end{aligned}
$$$$
E\left[w_{2 \alpha}(x, \infty)\right]
$$$$
=\frac{E\left[p_{\alpha}\right]_{\alpha}}{L} \sum_{n=1}^{\infty}\left(\frac{1-(-1)^{n}}{n \pi / L}\right)
$$$$
\times\left\{\frac{1}{E\left[E_{0 \alpha}\right] I(n \pi / L)^{4}-N(n \pi / L)^{2}}\right.
$$$$
\times\left[1+\frac{(n \pi / L)^{8} I^{2} \sigma_{E_{0}}^{2}}{\left\{E\left[E_{0 \alpha}\right] I(n \pi / L)^{4}-N(n \pi / L)^{2}\right\}^{2}}\right]
$$$$
-\frac{1}{E\left[E_{0 \alpha}\right] I(n \pi / L)^{4}-\left(N-2 k_{1}\right)(n \pi / L)^{2}+2 k_{0}}
$$$$
\times\left[1+\left(\left(\frac{n \pi}{L}\right)^{8} I^{2} \sigma_{E_{0}}^{2}\right)\right.
$$$$
\times\left(\left\{E\left[E_{0 \alpha}\right] I\left(\frac{n \pi}{L}\right)^{4}-\left(N-2 k_{1}\right)\left(\frac{n \pi}{L}\right)^{2}\right.\right.
$$$$
\left.\left.\left.\left.+2 k_{0}\right\}^{2}\right)^{-1}\right]\right\} \sin \frac{n \pi x}{L}
$$

where $\sigma_{E_{0}}^{2}$ is the variance of the Young modulus.

Formula (13) gives the variance of the system displacement for the steady-state vibrations $(t \rightarrow \infty)$ in the form

$$
\begin{aligned}
& \sigma_{w_{i \alpha}}^{2}(x, \infty) \\
& \quad=C_{w_{i \alpha}}(x, x, \infty, \infty) \\
& \quad=\sigma_{p_{\alpha}}^{2} \int_{0}^{L} \int_{0}^{\infty} E\left[H_{i \alpha}^{2}(x, \xi, \tau)\right] d \tau d \xi+E^{2}\left[p_{\alpha}\right]_{\alpha}
\end{aligned}
$$

$$
\begin{aligned}
\times \int_{0}^{L} \int_{0}^{L} \int_{0}^{\infty} \int_{0}^{\infty} C_{\left(H_{i} H_{i}\right)_{\alpha}} \\
\quad \times\left(x, x, \xi_{1}, \xi_{2}, \tau_{1}, \tau_{2}\right) d \tau_{1} d \tau_{2} d \xi_{1} d \xi_{2}
\end{aligned}
$$

where

$$
\begin{aligned}
& \sigma_{p_{\alpha}}^{2} \int_{0}^{L} \int_{0}^{\infty} E\left[H_{1 \alpha}^{2}(x, \xi, \tau)\right] d \tau d \xi \\
& =\frac{\sigma_{p_{\alpha}}^{2} L}{2(m L)^{2}} \\
& \times \sum_{n=1}^{\infty} E\left\{J_{I n \alpha}+J_{I I n \alpha}+J_{I, I I n \alpha}\right\} \sin ^{2} \frac{n \pi x}{L}, \\
& \sigma_{p_{\alpha}}^{2} \int_{0}^{L} \int_{0}^{\infty} E\left[H_{2 \alpha}^{2}(x, \xi, \tau)\right] d \tau d \xi \\
& =\frac{\sigma_{p_{\alpha}}^{2} L}{2(m L)^{2}} \\
& \times \sum_{n=1}^{\infty} E\left\{J_{I n \alpha}+J_{I I n \alpha}-J_{I, I I n \alpha}\right\} \sin ^{2} \frac{n \pi x}{L}, \\
& J_{\text {In } \alpha}=\frac{1}{4 \beta} E\left[\frac{1}{\omega_{\text {In } \alpha}^{2}}\right] \\
& =\frac{m}{4 \beta\left\{(n \pi / L)^{4} E\left[E_{0 \alpha}\right] I-(n \pi / L)^{2} N\right\}} \\
& \times\left[1+\left(\left(\frac{n \pi}{L}\right)^{8} I^{2} v_{E_{0 \alpha}}^{2}\right)\right. \\
& \left.\times\left(\left[\left(\frac{n \pi}{L}\right)^{4} I-\left(\frac{n \pi}{L}\right)^{2} \frac{N}{E\left[E_{0 \alpha}\right]}\right]^{2}\right)^{-1}\right], \\
& J_{I I n \alpha}=\frac{1}{4 \beta} E\left[\frac{1}{\omega_{I I n \alpha}^{2}}\right] \\
& =\frac{m}{4 \beta\left\{(n \pi / L)^{4} E\left[E_{0 \alpha}\right] I-(n \pi / L)^{2}\left(N-2 k_{1}\right)+2 k_{0}\right\}} \\
& \times\left[1+\left(\left(\frac{n \pi}{L}\right)^{8} I^{2} v_{E_{0 \alpha}}^{2}\right)\right. \\
& \times\left(\left\{\left(\frac{n \pi}{L}\right)^{4} E\left[E_{0 \alpha}\right] I-\left(\frac{n \pi}{L}\right)^{2}\right.\right. \\
& \left.\left.\left.\times \frac{\left(N-2 k_{1}\right)}{E\left[E_{0 \alpha}\right]}+\frac{2 k_{0}}{E\left[E_{0 \alpha}\right]}\right\}^{2}\right)^{-1}\right] \\
& J_{I, I I n \alpha}=8 \beta E\left[\frac{1}{\left(2 \beta^{2}+\omega_{I n \alpha}^{2}+\omega_{I I n \alpha}^{2}\right)^{2}-4 \bar{\omega}_{I n \alpha}^{2} \bar{\omega}_{I I n \alpha}^{2}}\right] .
\end{aligned}
$$


The numerical calculations have been done under the following assumptions.

(i) The mean values $E\left[E_{0 \alpha}\right]$ and $E\left[p_{\alpha}\right]$ are fuzzy numbers with triangular membership function (Figures 2 and 3),

(ii) The variation coefficients $v_{E_{0}}$ are constant and do not depend on the level of fuzziness.

(iii) Other quantities describing the structure are deterministic.

The following values of the parameters are used in the following numerical calculations: $L=10 \mathrm{~m}, E\left[E_{01}\right]=1$. $10^{10} \mathrm{Nm}^{-2}, E\left[E_{00 l}\right]=0,8 \cdot 10^{10} \mathrm{Nm}^{-2}, E\left[E_{00 r}\right]=1,2$. $10^{10} \mathrm{Nm}^{-2}, I=3,8 \cdot 10^{-4} \mathrm{~m}^{4}, k_{0}=1 \cdot 10^{5} \mathrm{Nm}^{2}, k_{1}=$ $2 \cdot 10^{4} \mathrm{Nm}^{2}, N=0$ and $N=0,4\left(\pi^{2} / L^{2}\right) E\left[E_{01}\right] I, v_{E_{0}}=0,2$, $E\left[p_{1}\right]=$ const., $E\left[p_{0 l}\right]=0,8 E\left[p_{1}\right]$, and $E\left[p_{0 r}\right]=1,2 E\left[p_{1}\right]$.

In (23) it has been assumed that $E^{2}\left[p_{\alpha}\right]_{\alpha}=0$.

In Figures 2, 3, 4, 5, 6, and 7 the membership of the expected value and variance of the beams response are presented.

\section{Fuzzy Stochastic Moving Load}

Let us notice that the general solution presented in Section 2 can also be used, after some modifications, if the doublebeam system is loaded by a fuzzy stochastic load moving with velocity $v$ (Figure 8 ).

In this case the right-hand side in (1) is equal to $p_{\alpha}(x, t)=$ $p_{\alpha}(x-v t)$.

The FRDIF $H_{i \alpha}(x, t)(i=1,2)$ in solutions (10)(16) should be replaced by fuzzy random dynamic moving influence function (FRDMIF) $H_{i m \alpha}(x, \xi, t)$ which satisfies, for $0 \leq t \leq L / v$, the equations

$$
\begin{gathered}
E_{0 \alpha} I \frac{\partial^{4} H_{\operatorname{Im} \alpha}(x, t)}{\partial x^{4}}+N \frac{\partial^{2} H_{\operatorname{Im} \alpha}(x, t)}{\partial x^{2}}+c_{\alpha} \frac{\partial H_{\operatorname{Im} \alpha}(x, t)}{\partial t} \\
+m_{\alpha} \frac{\partial^{2} H_{\operatorname{Im} \alpha}(x, t)}{\partial t^{2}}=\delta(x-v t), \\
E_{0 \alpha} I \frac{\partial^{4} H_{I \operatorname{Im} \alpha}(x, t)}{\partial x^{4}}+\left(N-2 k_{1 \alpha}\right) \frac{\partial^{2} H_{I \operatorname{Im} \alpha}(x, t)}{\partial x^{2}} \\
+2 k_{0 \alpha} H_{I \operatorname{Im} \alpha}(x, t)+c_{\alpha} \frac{\partial H_{I \operatorname{Im} \alpha}(x, t)}{\partial t} \\
+m_{\alpha} \frac{\partial^{2} H_{I \operatorname{Im} \alpha}(x, t)}{\partial t^{2}}=\delta(x-v t), \\
H_{1 m \alpha}(x, t)=\frac{H_{\operatorname{Im} \alpha}(x, t)+H_{I \operatorname{Im} \alpha}(x, t)}{2} \\
H_{2 m \alpha}(x, t)=\frac{H_{\operatorname{Im} \alpha}(x, t)-H_{I \operatorname{Im} \alpha}(x, t)}{2} .
\end{gathered}
$$

The fuzzy random dynamic moving influence functions have the forms

$$
\begin{aligned}
& H_{\operatorname{Im} \alpha}(x, t) \\
& =2 \frac{L^{3}}{E I_{0 \alpha}} \sum_{n=1}^{\infty} \frac{\sin n \pi(v t / L) \sin n \pi(x / L)}{(n \pi)^{2}\left[(n \pi)^{2}-N_{0}-\eta^{2}\right]}-2 \frac{L^{3}}{E I_{0 \alpha}} \eta \\
& \times \sum_{n=1}^{\infty} \frac{\sin \left[\left(n \pi \sqrt{(n \pi)^{2}-N_{0}}\right) / \eta\right](v t / L) \sin n \pi(x / L)}{(n \pi)^{2}\left[(n \pi)^{2}-N_{0}-\eta^{2}\right] \sqrt{(n \pi)^{2}-N_{0}}} \\
& H_{I \operatorname{Im} \alpha}(x, t) \\
& =2 \frac{L^{3}}{E I_{0 \alpha}} \sum_{n=1}^{\infty} \frac{\sin n \pi(v t / L) \sin n \pi(x / L)}{(n \pi)^{4}-(n \pi)^{2}\left[N_{0}-2 \kappa_{1}+\eta^{2}\right]+2 \kappa_{0}}-2 \frac{L^{3}}{E I_{0 \alpha}} \eta \\
& \times \sum_{n=1}^{\infty}\left(\left((n \pi) \sin \left[\frac{\sqrt{(n \pi)^{4}-(n \pi)^{2}\left(N_{0}-2 \kappa_{1}\right)+2 \kappa_{0}}}{\eta} \frac{v t}{L}\right]\right.\right. \\
& \left.\times \sin n \pi \frac{x}{L}\right) \\
& \times\left(\left\{(n \pi)^{4}-(n \pi)^{2}\left[N_{0}-2 \kappa_{1}+\eta^{2}\right]+2 \kappa_{0}\right\}\right. \\
& \left.\left.\times \sqrt{(n \pi)^{4}-(n \pi)^{2}\left(N_{0}-2 \kappa_{1}\right)+2 \kappa_{0}}\right)^{-1}\right)
\end{aligned}
$$

where $N_{0}=N L^{2} / E I_{0 \alpha}, \kappa_{0}=k_{0} L^{4} / E I_{0 \alpha}, \kappa_{1}=k_{1} L^{2} / E I_{0 \alpha}$, and $\eta^{2}=m v^{2} L^{2} / E I_{0 \alpha}$.

The general solutions for moving fuzzy stochastic load after modification of (10)-(16) have the forms

$$
w_{i m \alpha}(x, t)=\int_{0}^{t} H_{i m \alpha}(x, t-\tau) p_{\alpha}(\tau) d \tau
$$

where $i=1,2,0 \leq t \leq L / v$,

$$
E\left[w_{i m \alpha}(x, t)\right]_{\alpha}=\int_{0}^{t} E\left[H_{i m \alpha}(x, t-\tau)\right] E\left[p_{\alpha}(\tau)\right] d \tau,
$$




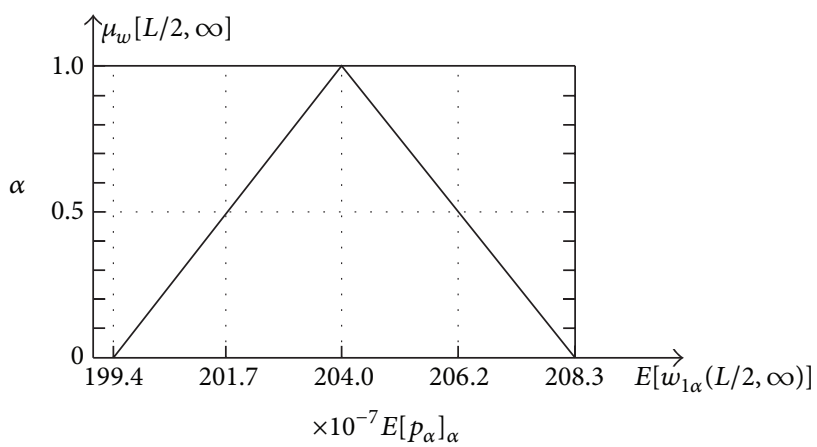

(a)

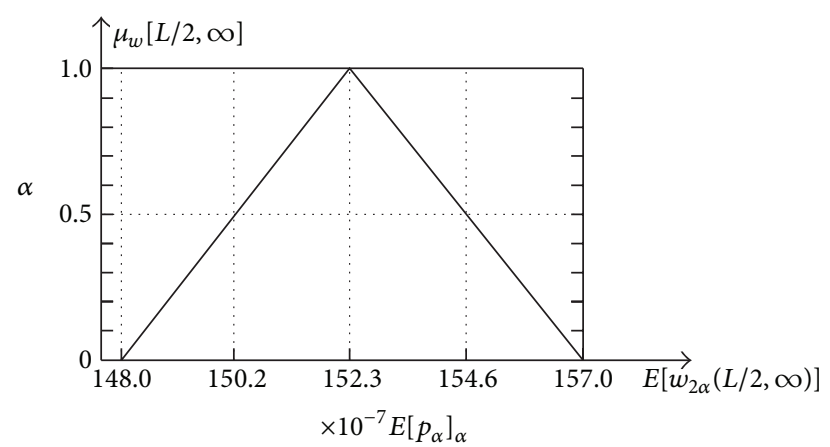

(b)

Figure 2: Membership function of the mean value for $N=0$.

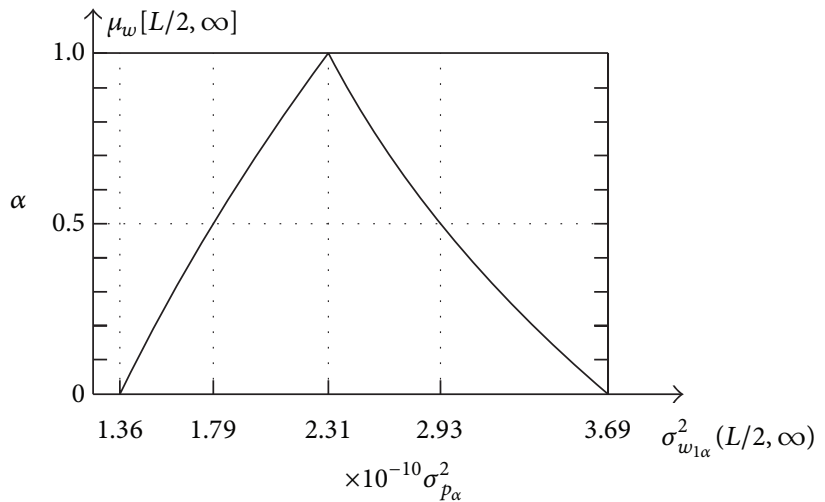

(a)

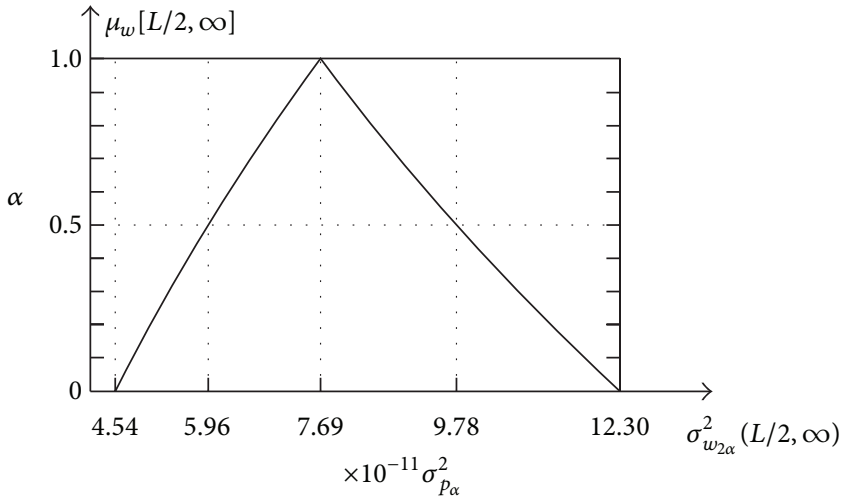

(b)

FIgURE 3: Membership function of the variance for $N=0$.

$$
\begin{aligned}
& E\left[w_{\text {im } \alpha}(x, t)\right]_{\alpha l} \\
& \quad=\min \left\{\int_{0}^{t} E\left[H_{i m \alpha}(x, t-\tau)\right] E\left[p_{\alpha}(\tau)\right] d \tau\right\},
\end{aligned}
$$

$$
\begin{aligned}
& E\left[w_{\text {im } \alpha}(x, t)\right]_{\alpha r} \\
& =\max \left\{\int_{0}^{t} E\left[H_{\text {im } \alpha}(x, t-\tau)\right] E\left[p_{\alpha}(\tau)\right] d \tau\right\},
\end{aligned}
$$

$$
\begin{gathered}
C_{w_{i m \alpha}}\left(x_{1}, x_{2}, t_{1}, t_{2}\right) \\
=\int_{0}^{t_{1}} \int_{0}^{t_{2}} E\left[H_{i m \alpha}\left(x_{1}, t_{1}-\tau_{1}\right) H_{i m \alpha}\left(x_{2}, t_{2}-\tau_{2}\right)\right] \\
\quad \times C_{(p p)_{\alpha}}\left(\tau_{1}, \tau_{2}\right) d \tau_{1} d \tau_{2}
\end{gathered}
$$$$
+\int_{0}^{t_{1}} \int_{0}^{t_{2}} C_{\left(H_{i} H_{i}\right)_{\alpha}}\left(x_{1}, x_{2}, t_{1}-\tau_{1}, t_{2}-\tau_{2}\right)
$$$$
\times E\left[p_{\alpha}\left(\xi_{1}, \tau_{1},\right)\right] E\left[p_{\alpha}\left(\xi_{2}, \tau_{2},\right)\right] d \tau_{1} d \tau_{2},
$$

where

$$
\begin{aligned}
& C_{\left(H_{i} H_{j}\right)_{\alpha}}\left(x_{1}, x_{2}, t_{1}, t_{2}\right) \\
& =E\left[H_{i m \alpha}\left(x_{1}, t_{1}\right) H_{j m \alpha}\left(x_{2}, t_{2}\right)\right]_{\alpha} \\
& \quad-E\left[H_{i m \alpha}\left(x_{1}, t_{1}\right)\right]_{\alpha} E\left[H_{j m \alpha}\left(x_{2}, t_{2}\right)\right]_{\alpha}, \\
& C_{(p p)_{\alpha}}\left(\tau_{1}, \tau_{2}\right) \\
& =E\left[p_{\alpha}\left(\tau_{1}\right) p_{\alpha}\left(\tau_{2}\right)\right]_{\alpha} \\
& \quad-E\left[p_{\alpha}\left(\tau_{1}\right)\right]_{\alpha} E\left[p_{\alpha}\left(\tau_{2}\right)\right]_{\alpha} .
\end{aligned}
$$

The lower and upper endpoints of the covariance could be defined using (32) as

$$
\begin{aligned}
& C_{w_{i \alpha m l}}\left(x_{1}, x_{2}, t_{1}, t_{2}\right) \\
& =\min \left\{\int_{0}^{t_{1}} \int_{0}^{t_{2}} E\left[H_{i m \alpha}\left(x_{1}, t_{1}-\tau_{1}\right) H_{i m \alpha}\left(x_{2}, t_{2}-\tau_{2}\right)\right]\right. \\
& \times C_{(p p)_{\alpha}}\left(\tau_{1}, \tau_{2}\right) d \tau_{1} d \tau_{2}
\end{aligned}
$$




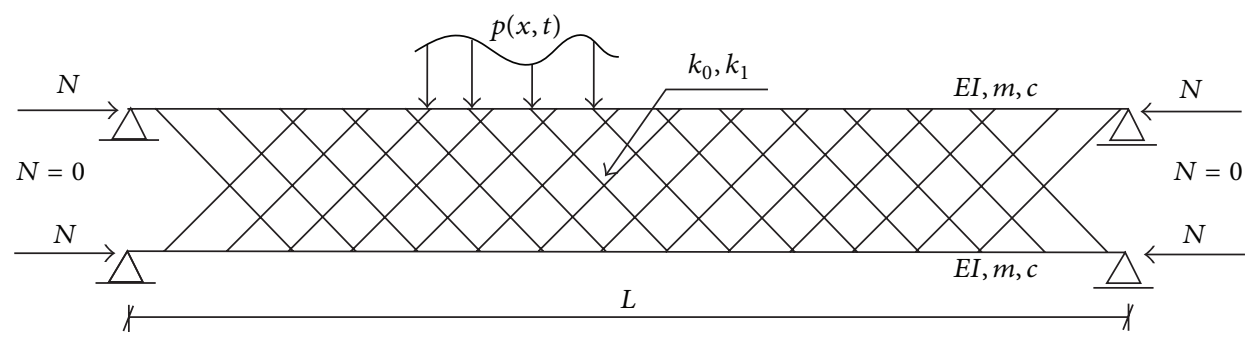

(a)

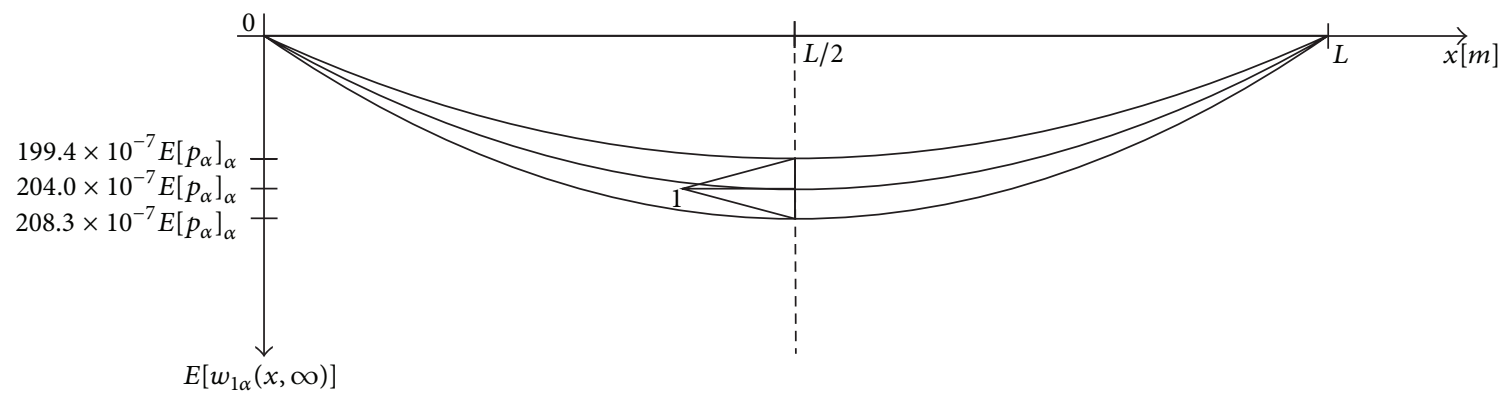

(b)

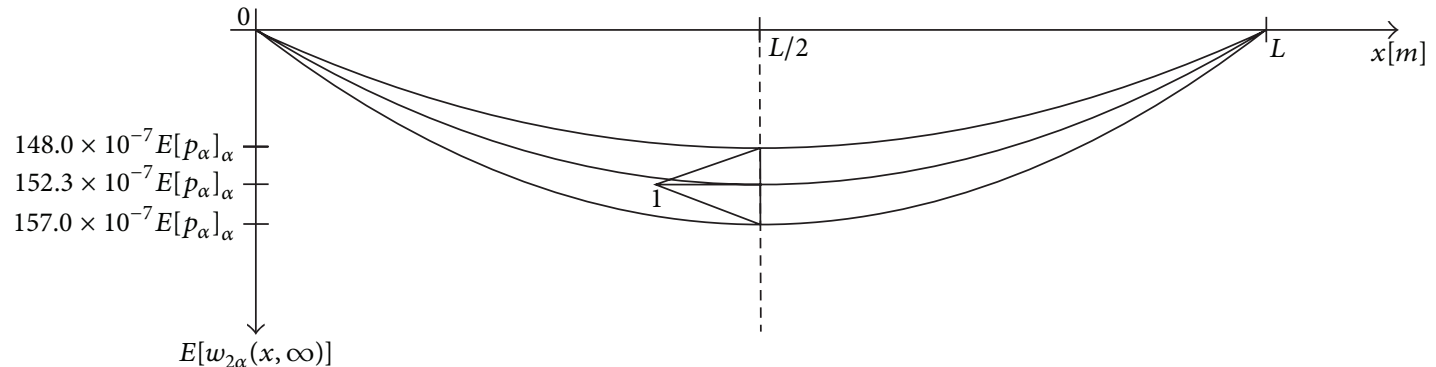

(c)

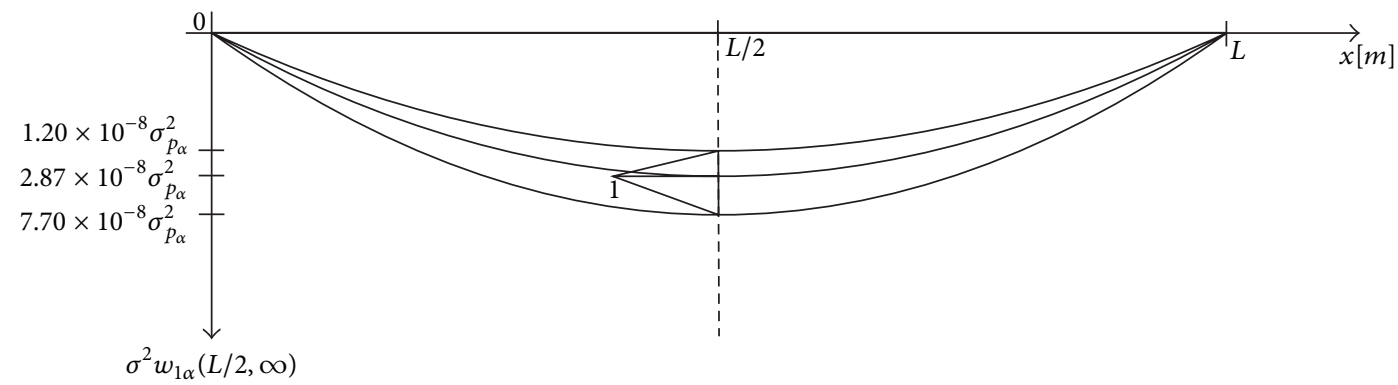

(d)

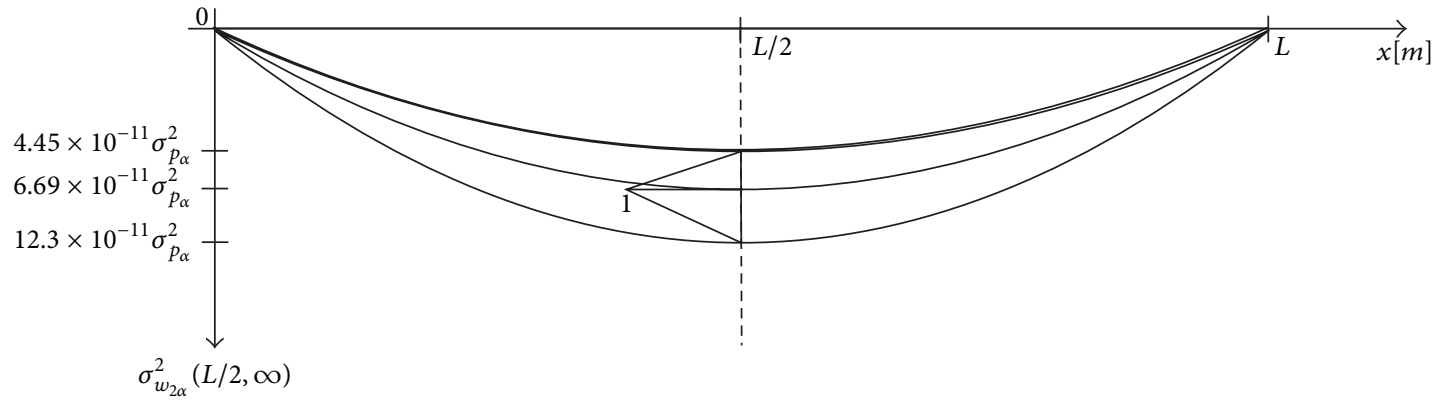

(e)

FIGURE 4: Expected value and variance of the response of the beams. 


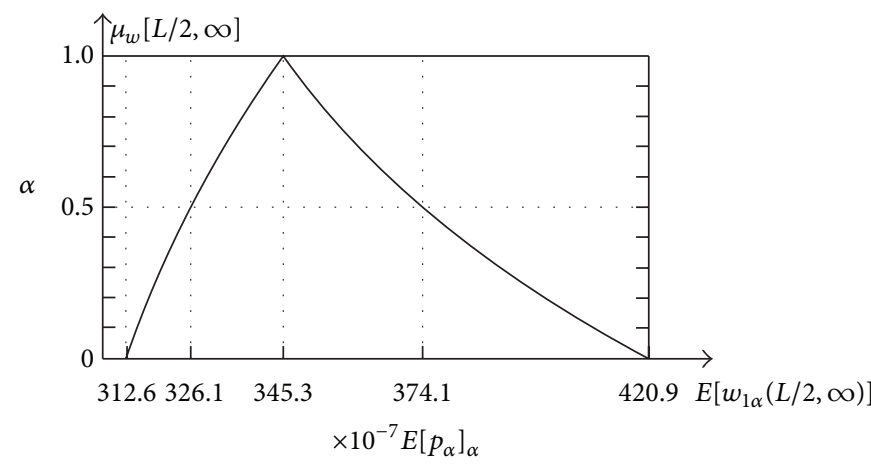

(a)

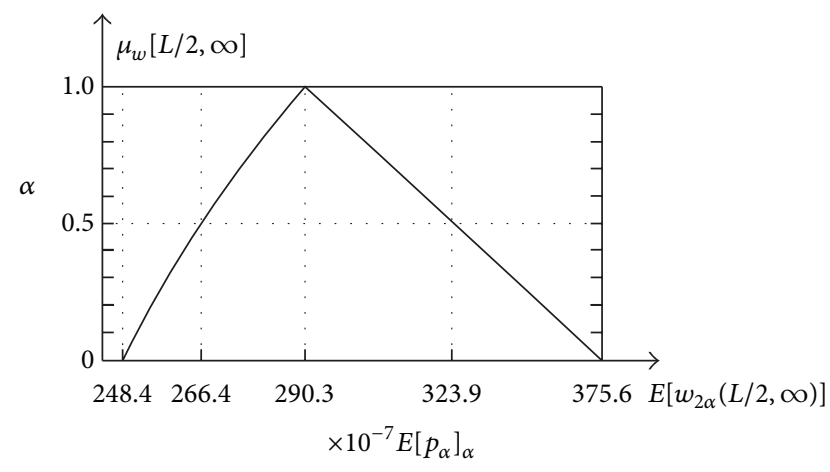

(b)

Figure 5: Membership function of the mean value for $N=0,4\left(\pi^{2} / L^{2}\right) E\left[E_{01}\right] I$.

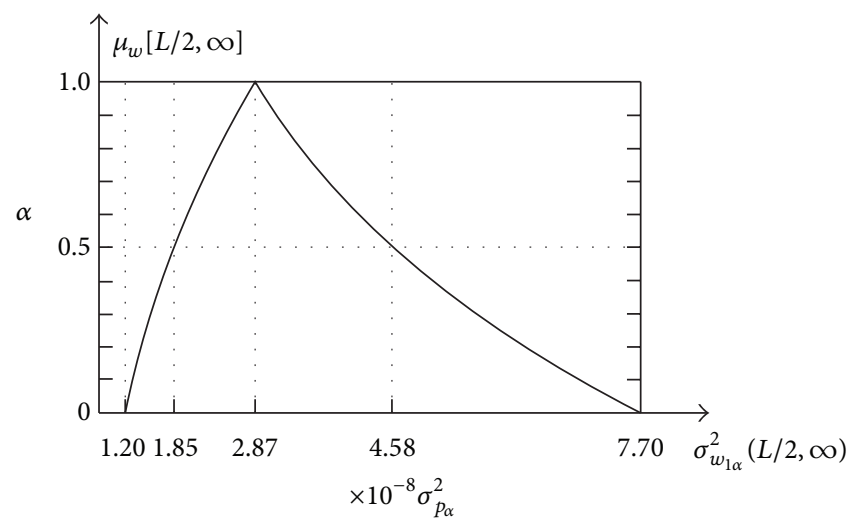

(a)

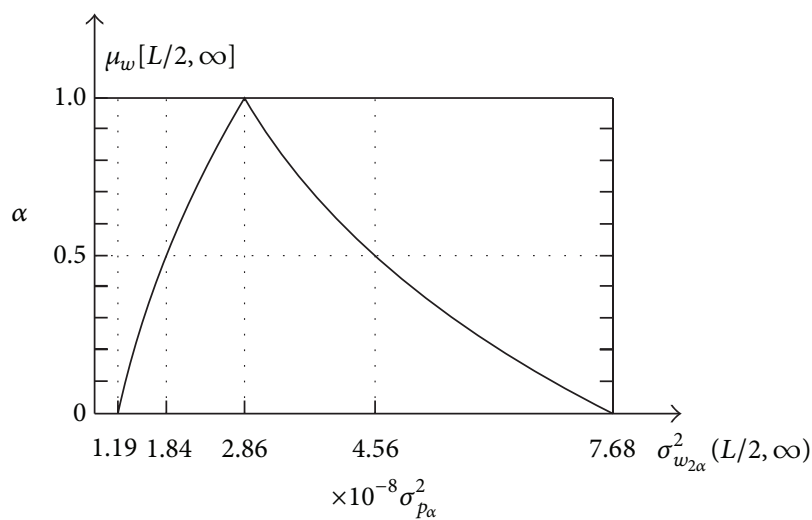

(b)

FIGURE 6: Membership function of the mean variance for $N=0,4\left(\pi^{2} / L^{2}\right) E\left[E_{01}\right] I$.

$$
\begin{gathered}
+\int_{0}^{t_{1}} \int_{0}^{t_{2}} C_{\left(H_{i} H_{i}\right)_{\alpha}}\left(x_{1}, x_{2}, t_{1}-\tau_{1}, t_{2}-\tau_{2}\right) \\
\times E\left[p_{\alpha}\left(\xi_{1}, \tau_{1},\right)\right] \\
\left.\times E\left[p_{\alpha}\left(\xi_{2}, \tau_{2},\right)\right] d \tau_{1} d \tau_{2}\right\}, \\
C_{w_{i \alpha m r}}\left(x_{1}, x_{2}, t_{1}, t_{2}\right) \\
=\max \left\{\int_{0}^{t_{1}} \int_{0}^{t_{2}} E\left[H_{i m \alpha}\left(x_{1}, t_{1}-\tau_{1}\right) H_{i m \alpha}\left(x_{2}, t_{2}-\tau_{2}\right)\right]\right. \\
\times C_{(p p)_{\alpha}}\left(\tau_{1}, \tau_{2}\right) d \tau_{1} d \tau_{2} \\
+\int_{0}^{t_{1}} \int_{0}^{t_{2}} C_{\left(H_{i} H_{i}\right)_{\alpha}}\left(x_{1}, x_{2}, t_{1}-\tau_{1}, t_{2}-\tau_{2}\right) \\
\left.\times E\left[p_{\alpha}\left(\tau_{1}\right)\right] E\left[p_{\alpha}\left(\tau_{2}\right)\right] d \tau_{1} d \tau_{2}\right\},
\end{gathered}
$$

where $0 \leq t_{1}$ and $t_{2} \leq L / v$.
For steady-state solutions and it should be assumed that $t_{1}=t_{2}=L / v$.

\section{Conclusion}

In the paper, the dynamic behavior of a double EulerBernoulli beam complex system with uncertain parameters (fuzzy random variables) under a fuzzy stochastic excitation and axial compression has been studied. The load process is fuzzy random both in space and time. In order to find the solution for the membership function of the probabilistic characteristics of the response of the structure, the idea of the fuzzy random dynamic influence function has been used. The probabilistic characteristics of the response of the structure are sought in the form of the first two probabilistic moments, that is, the expected value and the correlation (covariance) function. This double Euler-Bernoulli beam system can be also treated as a theoretical model of a sandwich beam. The algorithm (similar to the one presented in the paper for the double Euler-Bernoulli beam complex system) can be used in stochastic dynamic analysis of other complex systems like strings, beams, plates, and so on, with uncertain parameters. 


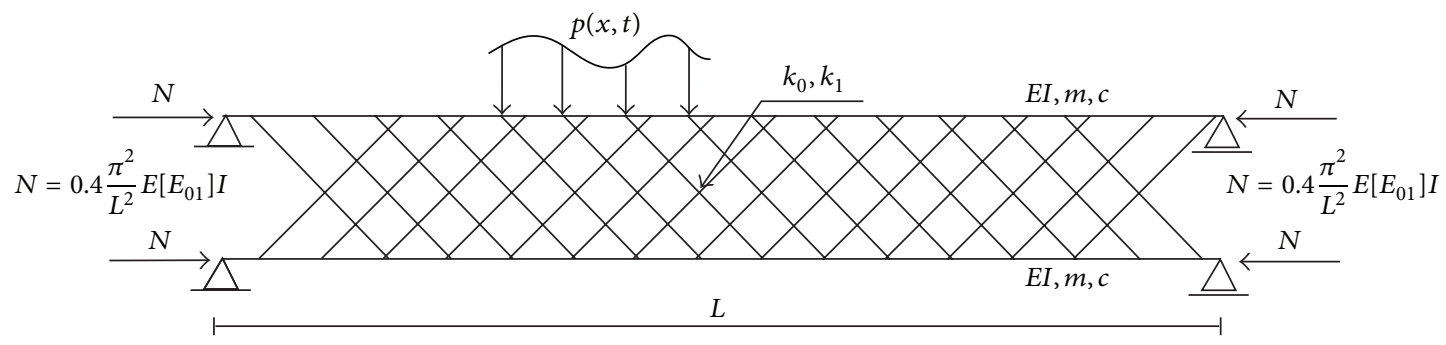

(a)

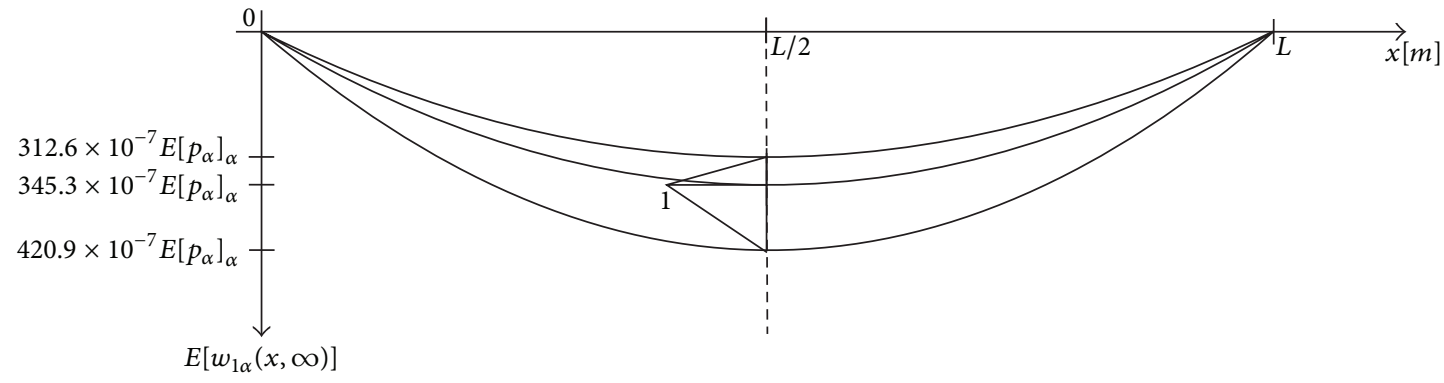

(b)

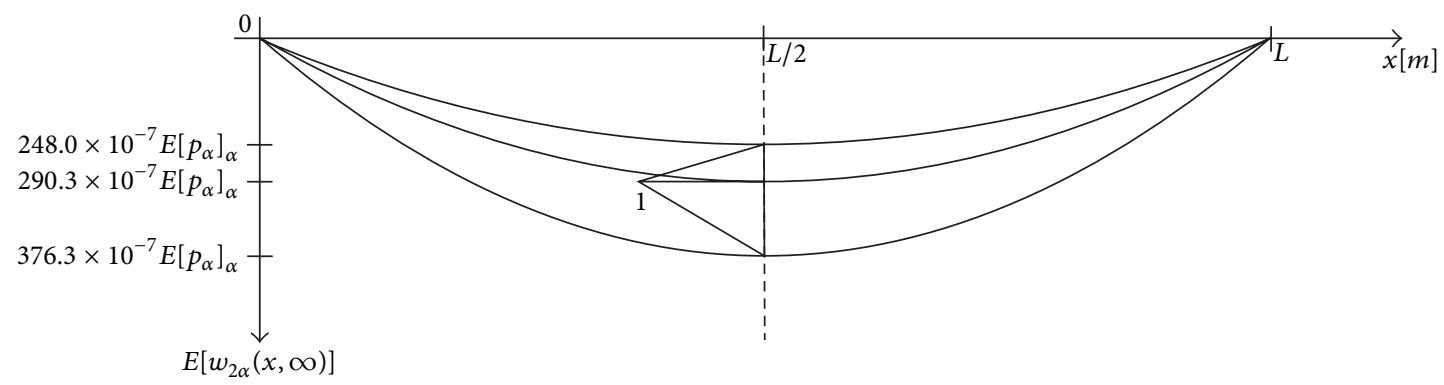

(c)

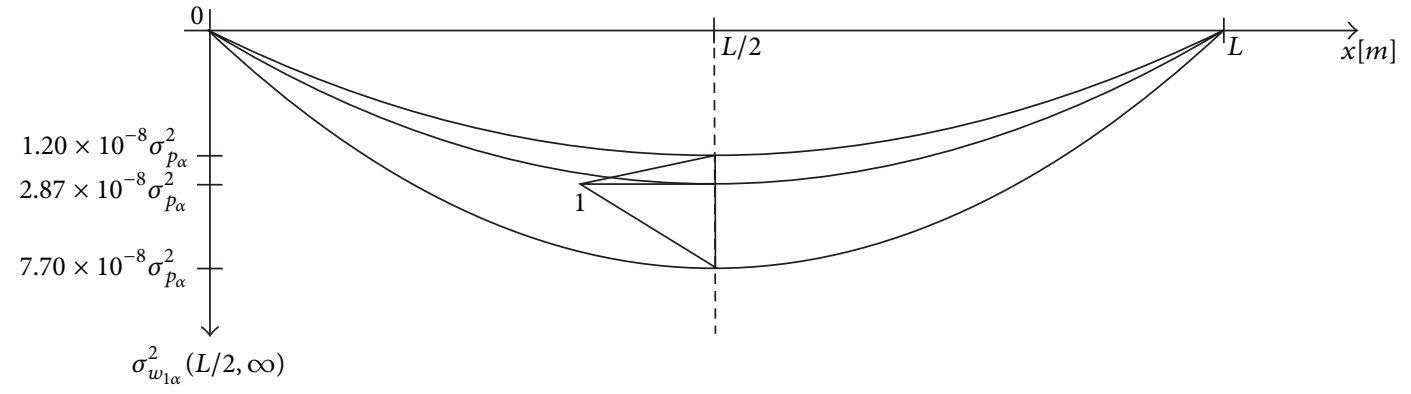

(d)

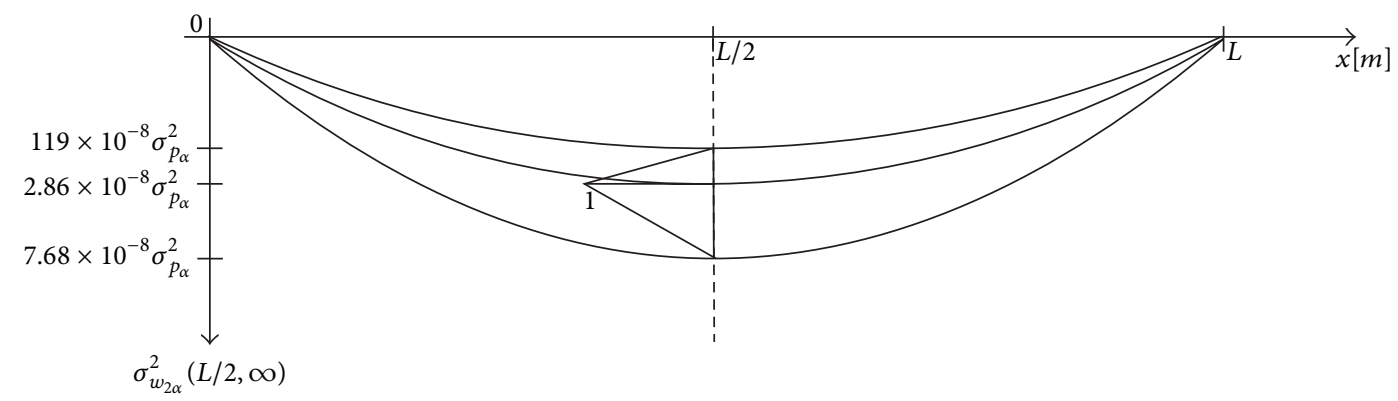

(e)

FIGURE 7: Expected value and variance of the response of the beams. 


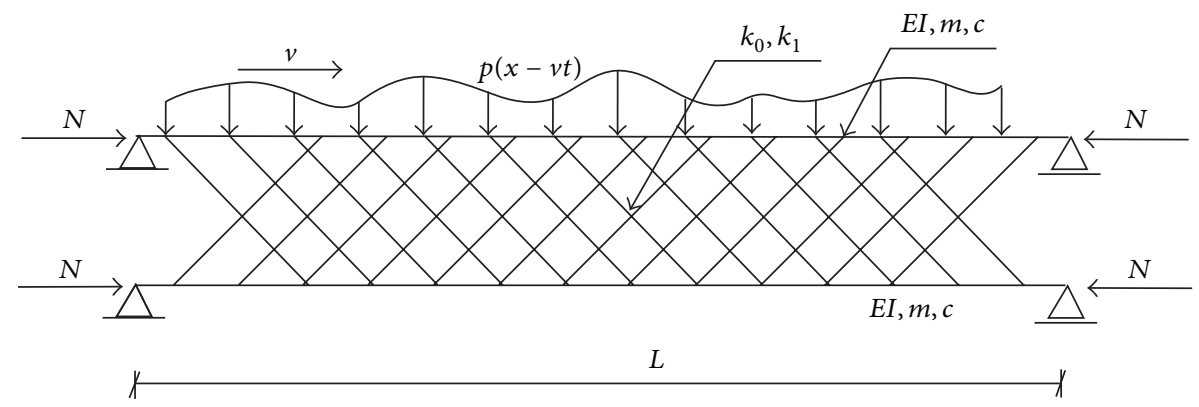

Figure 8: The double-beam system loaded by fuzzy stochastic moving load.

\section{Acknowledgment}

The second author gratefully acknowledges the support of the Polish National Science Centre under Grant number ID: 147573.

\section{References}

[1] L. A. Zadeh, "Fuzzy sets," Information and Computation, vol. 8, pp. 338-353, 1965.

[2] H. Kwakernaak, "Fuzzy random variables. I. Definitions and theorems," Information Sciences, vol. 15, no. 1, pp. 1-29, 1978.

[3] M. L. Puri and D. A. Ralescu, "Fuzzy random variables," Journal of Mathematical Analysis and Applications, vol. 114, no. 2, pp. 409-422, 1986.

[4] B. Möller and M. Beer, Fuzzy Randomness. Uncertainty in civil engineering and computational mechanics, Springer, Berlin, Germany, 2004.

[5] Z. Oniszczuk, "Transverse vibrations of elastically connected double-string complex system, Part I: free vibrations," Journal of Sound and Vibration, vol. 232, no. 2, pp. 355-366, 2000.

[6] Z. Oniszczuk, "Transverse vibrations of elastically connected double-string complex system, Part II: forced vibrations," Journal of Sound and Vibration, vol. 232, no. 2, pp. 367-386, 2000.

[7] Z. Oniszczuk, "Free transverse vibrations of elastically connected simply supported double-beam complex system," Journal of Sound and Vibration, vol. 232, no. 2, pp. 387-403, 2000.

[8] Z. Oniszczuk, "Damped vibration analysis of an elastically connected complex double-string system," Journal of Sound and Vibration, vol. 264, no. 2, pp. 253-271, 2003.

[9] Z. Oniszczuk, "Forced transverse vibrations of an elastically connected complex simply supported double-beam system," Journal of Sound and Vibration, vol. 264, no. 2, pp. 273-286, 2003.

[10] S. Kukla, "Free vibration of the system of two beams connected by many translational springs," Journal of Sound and Vibration, vol. 172, no. 1, pp. 130-135, 1994.

[11] H. V. Vu, A. M. Ordóñez, and B. H. Karnopp, "Vibration of a double-beam system," Journal of Sound and Vibration, vol. 229, no. 4, pp. 807-822, 2000.

[12] Y. Q. Zhang, Y. Lu, S. L. Wang, and X. Liu, "Vibration and buckling of a double-beam system under compressive axial loading," Journal of Sound and Vibration, vol. 318, no. 1-2, pp. 341-352, 2008.
[13] W. Szcześniak, "Vibration of elastic sandwich and elastically connected double-beam system under moving loads," Scientific Works of Warsaw University of Technology, Civil Engineering, vol. 132, pp. 111-151, 1998 (Polish).

[14] M. Abu-Hilal, "Dynamic response of a double Euler-Bernoulli beam due to a moving constant load," Journal of Sound and Vibration, vol. 297, no. 3-5, pp. 477-491, 2006.

[15] J. Rusin, P. Śniady, and P. Śniady, "Vibrations of double-string complex system under moving forces. Closed solutions," Journal of Sound and Vibration, vol. 330, no. 3, pp. 404-415, 2011.

[16] G. Adomian, Stochastic systems, vol. 169, Academic Press, Orlando, Fla, USA, 1983.

[17] P. Śniady, R. Adamowski, G. Kogut, and W. ZielichowskiHaber, "Spectral stochastic analysis of structures with uncertain parameters," Probabilistic Engineering Mechanics, vol. 23, no. 1, pp. 76-83, 2008.

[18] K. Mazur-Śniady, P. Śniady, and W. Zielichowski-Haber, "Dynamic response of micro-periodic composite rods with uncertain parameters under moving random load," Journal of Sound and Vibration, vol. 320, no. 1-2, pp. 273-288, 2009.

[19] R. G. Ghanem and P. D. Spanos, Stochastic Finite Elements: A Spectral Approach, Springer, New York, NY, USA, 1991.

[20] W. K. Liu, A. Mani, and T. Belytschko, "Finite element methods in probabilistic mechanics," Probabilistic Engineering Mechanics, vol. 2, no. 4, pp. 201-213, 1987.

[21] J. Li and S. Liao, "Response analysis of stochastic parameter structures under non-stationary random excitation," Computational Mechanics, vol. 27, no. 1, pp. 61-68, 2001.

[22] W. Mironowicz and P. Śniady, "Dynamics of machine foundations with random parameters," Journal of Sound and Vibration, vol. 112, no. 1, pp. 23-30, 1987.

[23] H. Jensen and W. D. Iwan, "Response of systems with uncertain parameters to stochastic excitation," Journal of Engineering Mechanics, vol. 118, no. 5, pp. 1012-1025, 1992.

[24] I. D. Gupta and R. G. Joshi, "Response spectrum superposition for structures with uncertain properties," Journal of Engineering Mechanics, vol. 127, no. 3, pp. 233-241, 2001.

[25] F. Yamazaki, M. Shinozuka, and G. Dasgupta, "Neumann expansion for stochastic finite element analysis," Journal of Engineering Mechanics, vol. 114, no. 8, pp. 1335-1354, 1988.

[26] C. Soize, "Vibration damping in low-frequency range due to structural complexity: a model based on the theory of fuzzy structures and model parameters estimation," Computers of Structures, vol. 58, no. 5, pp. 901-915, 1996. 
[27] M. Gładysz and P. Śniady, "Spectral density of the bridge beam response with uncertain parameters under a random train of moving forces," Archives of Civil and Mechanical Engineering, vol. 9, no. 3, pp. 31-47, 2009.

[28] P. Sniady, K. Mazur-Sniady, R. Sieniawska, and S. Zukowski, "Fuzzy stochastic elements method: spectral approach," Mechanical Systems and Signal Processing, vol. 37, pp. 152-162, 2013. 


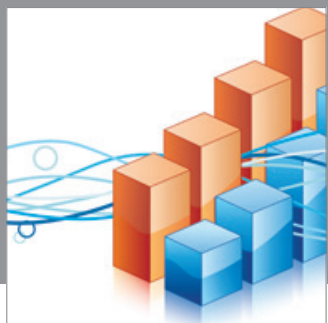

Advances in

Operations Research

mansans

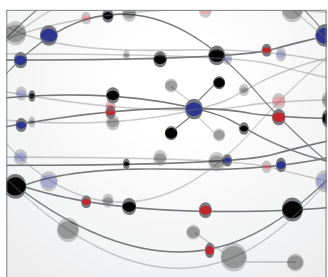

The Scientific World Journal
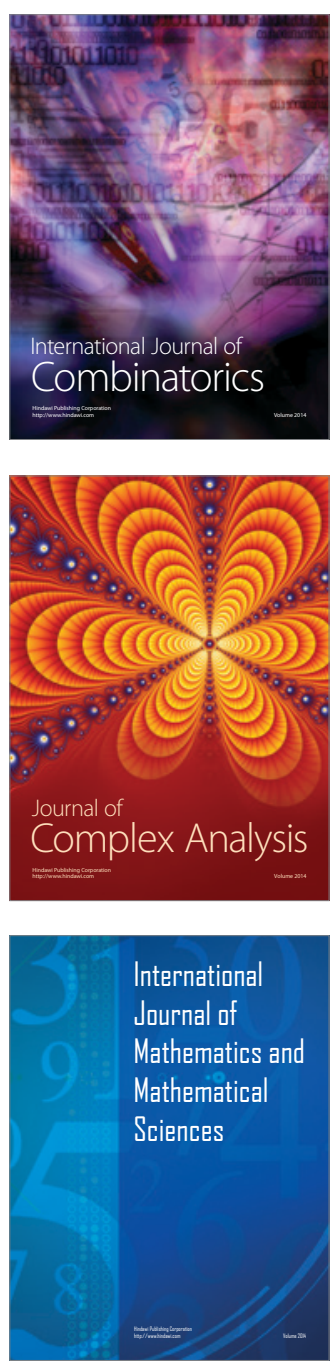
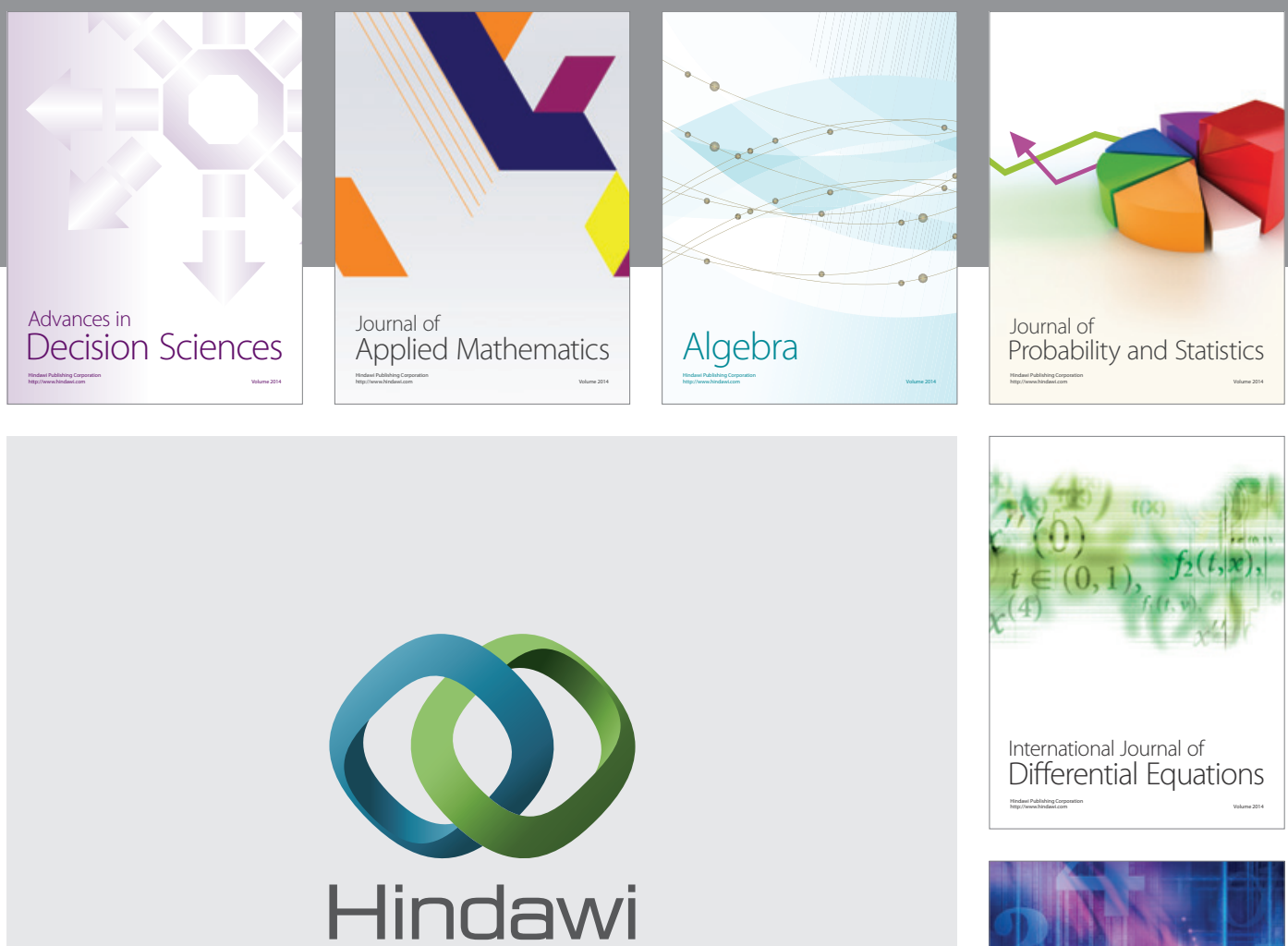

Submit your manuscripts at http://www.hindawi.com
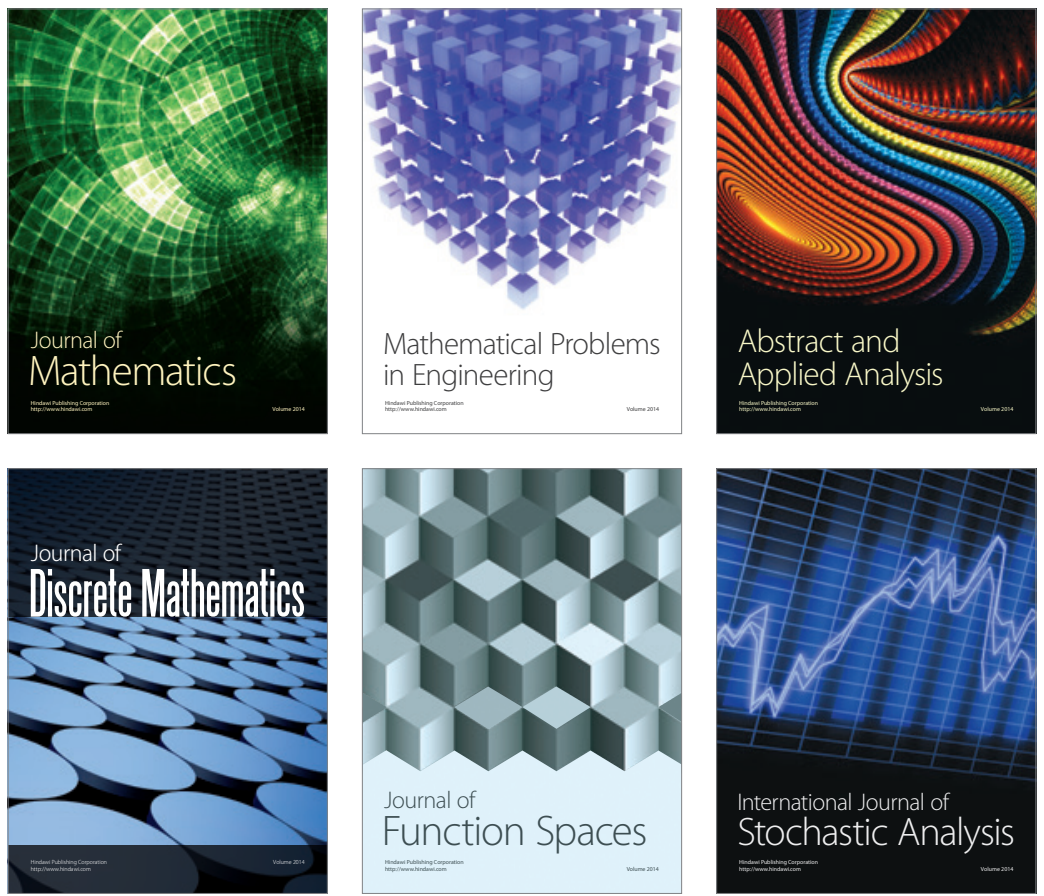

Journal of

Function Spaces

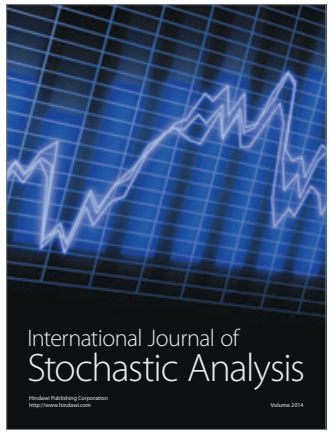

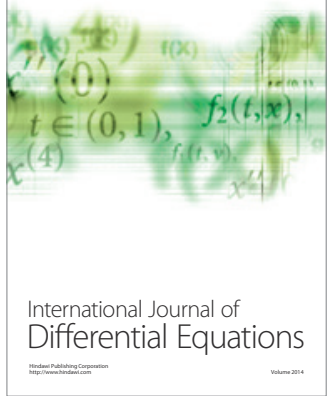
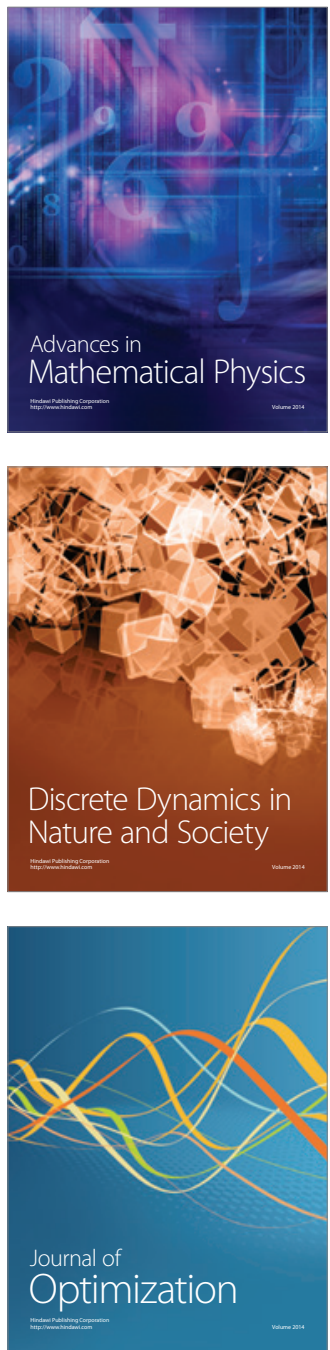